\title{
THE CARLESON MEASURE AND MEROMORPHIC FUNCTIONS OF UNIFORMLY BOUNDED CHARACTERISTIC
}

\author{
Ž. Pavićević
}

\begin{abstract}
For a meromorphic function $f(z)$ defined in the unit disc $D:|z|<1$ on the complex $z$-plane, $z=x+i y$, we denote its spherical derivative by $f^{\#}(z)$ and introduce the differentiable form $d \mu_{f}(z)=\left(1-|z|^{2}\right)\left[f^{\#}(z)\right]^{2} d x d y$. We prove that $f(z)$ has the uniformly bounded characteristic if and only if the measure $\mu_{f}(z)$ is the Carleson measure. This result answers a question posed by S. Yamashita in Internat. J. Math. Math. Sci. 8, 1985, pp. 477-482.
\end{abstract}

1. Let $f(z)$ be a meromorphic function defined in the unit disc $D:|z|<1$ on the complex $z$-plane, $z=x+i y$, and $f^{\#}(z)=\left|f^{\prime}(z)\right|\left[1+|f(z)|^{2}\right]^{-1}$ its spherical derivative. For an arbitrary $w \in D$ and $t, 0<t<1$, we denote $\varphi_{w}(z)=(z-w)(1-\bar{w} z)^{-1}$ and $\Delta(w, t)=\left\{z \in D ;\left|\varphi_{w}(z)\right|<t\right\}$.

The Nevanlinna characteristic function $T(r, f)$ of the function $f(z)$ is defined by the Ahlfors-Shimizu formula

$$
T(r, f)=\frac{1}{\pi} \int_{0}^{r} \frac{S(t, f)}{t} d t, \quad 0<r<1
$$

where

$$
S(t, f)=\iint_{|z|<t}\left[f^{\#}(z)\right]^{2} d x d y, \quad 0<t<1 .
$$

In [5], S. Yamashita proved that the Nevanlinna characteristic $T(r, w, f)$ of the function $f(w+(1-|w|) z), w \in D$ fixed, is of the form

$$
T(r, w, f)=\frac{1}{\pi} \int_{0}^{r} \frac{1}{t}\left[\iint_{D(w, t)}\left[f^{\#}(z)\right]^{2} d x d y\right] d t, \quad 0<r<1,
$$

where $D(w, t)=\{z \in D ;|z-w|<t(1-|w|)\}, 0<t<1$. In particular, $T(r, 0, f)=T(r, f)$.

Denote $T(1, w, f)=\lim _{r \rightarrow 1-o} T(r, w, f)$. If $T(1, f)<+\infty$, then $f(z)$ belongs to the class $\mathrm{BC}$ of meromorphic functions with bounded Nevanlinna characteristic. If $\sup _{|z|<1}(1-|z|) f^{\#}(z)<+\infty$, then $f(z)$ belongs to the class $N$ of normal meromorphic functions in $D$ (cf. [2]).

For any $w \in D$, we denote $f_{w}(z)=f\left(\varphi_{w}(z)\right), \varphi_{w}(z)=(z-w)(1-\bar{w} z)^{-1}$. 

only if

Theorem 1. A meromorphic function $f(z)$ in $D$ belongs to $B C \cap N$ if and

$$
\sup _{|w|<1} T(1, w, f)<+\infty
$$

Proof. Let $f(z) \in \mathrm{BC} \cap N$. Since $f(z) \in \mathrm{BC}$, then, according to [4], p. 351, Theorem 2.1

$$
\sup _{|w|<\varrho} T\left(1, f_{w}\right) \leq c_{\varrho}<+\infty
$$

for any $\varrho, 0<\varrho<1$.

It immediately follows from the definition that

$$
T\left(1, f_{w}\right)=\frac{1}{\pi} \int_{0}^{1}\left[\iint_{\Delta(w, t)}\left[f^{\#}(z)\right]^{2} d x d y\right] d t
$$

(cf. [4]).

Since $D(w, t) \subset \Delta(w, t)$ for any $w \in D$ and $0<t<1$, then

$$
T(1, w, f) \leq T\left(1, f_{w}\right)
$$

for any $w \in D$.

It follows from (2) and (3) that

$$
\sup _{|w|<\varrho} T(1, w, f) \leq c_{\varrho}<+\infty
$$

for any $\varrho, 0<\varrho<1$.

Since $f \in N$, then, according to [5], Theorem 1

$$
\sup _{q<|w|<1} T(1, w, f) \leq c_{q}<+\infty
$$

for any $q, 0<q<1$.

Choosing $q<\varrho$, we obtain from (4) and (5)

$$
\sup _{|w|<1} T(1, w, f) \leq \max \left(c_{\varrho}, c_{q}\right)<+\infty
$$

and (1) is proved. 
Sufficiency. We put $w=0$ in (1). Then $T(1,0, f)=T(1, f)<+\infty$, so that $f(z) \in \mathrm{BC}$.

It follows from (1) that

$$
\sup _{q<|w|<1} T(1, w, f) \leq \sup _{|w|<1} T(1, w, f)<+\infty
$$

for every $q, 0<q<1$. Combining (6) with [5], Theorem 1, we conclude that $f(z) \in N$.

2. Following S. Yamashita ([4]), a meromorphic function $f(z)$ defined in $D$ is called a function with uniformly bounded characteristic if $\sup _{w \in D} T\left(1, f_{w}\right)$. The class of such functions is denoted by $\cup B C$. The inclusion $\cup B C \subset B C \cap N$, proved by S. Yamashita in [4], now follows immediately from our Theorem 1.

3. For a meromorphic function $f(z)$ defined in $D$ we introduce the differentiable form $d \mu_{f}(z)=\left(1-|z|^{2}\right)\left[f^{\#}(z)\right]^{2} d x d y$ and the measure $\mu_{f}(E)=$ $\iint_{E} d \mu_{f}(z)$ generated by $d \mu_{f}(z)$ on a Borel set $E \subset D$. Let

$$
Q\left(\mu_{f}, w\right)=\frac{1}{2 \bar{u}} \mu_{f}(R(w))(1-|w|)^{-1},
$$

where $R(w)=\{z \in D ;|w|<|z|<1,|\arg z-\arg w|<\pi(1-|w|)\}$ for $w \neq 0$, and $R(w)=D$ for $w=0$. The measure $\mu_{f}$ is called the Carleson measure if $\sup _{w \in D} Q\left(\mu_{f}, w\right)<+\infty$ (cf. [6], p. 38).

Theorem 2. A meromorphic function $f(z)$ belongs to the class $\cup B C$ if and only if $f(z)$ is normal and $\mu_{f}$ is the Carleson measure.

In fact, the necessity in Theorem 2 follows from [4], Theorem 3.1 and [6], Theorem 2. The sufficiency in Theorem 2 is contained in the proof of Theorem 3 from [6], pp. $42-43$.

4. In the paper [7], S. Yamashita posed the following problem: Does a meromorphic function $f(z)$ belong to the class $\cup B C$ if the measure $\mu_{f}$ is the Carleson measure?

The solution of this problem is contained in part 5 , Theorem 3 .

We prove the following

Lemma. If the measure $\mu_{f}$ for a meromophic function $f(z)$ in $D$ is the Carleson measure, $f(z)$ is normal (i.e. $f(z) \in N$ ).

Proof. Let $a, \frac{1}{4}<a<1$, be fixed. Then $\frac{1}{4}<|z-w||1-\bar{w} z|^{-1}$ for any $z \in D$, for which $|z|<r=r(a)=\frac{1}{5}(4 a-1)$, and for any $w$ with $a<|w|<1$. 

have

Since $\log \left(|z|^{-1}\right) \leq c\left(1-|z|^{2}\right)$ for $|z|>\frac{1}{4}$ (cf. for instance [1], p. 238), we

$$
\log |1-\bar{w} z||z-w|^{-1} \leq c\left(1-\left(|1-\bar{w} z|^{-1}|z-w|\right)^{2}\right)=c \frac{\left(1-|z|^{2}\right)\left(1-|w|^{2}\right)}{|1-\bar{w} z|^{2}}
$$

for all $z,|z|<r(a)$, and $w, a<|w|<1$, with $\frac{1}{4}<a<1$.

It is known ([4]) that

$$
T\left(r, f_{w}\right)=\iint_{|z|<r}\left[f^{\#}(z)\right]^{2} \log \left|\frac{1-\bar{w} z}{z-w}\right| d x d y, \quad 0<r \leq 1 .
$$

We hence have, for any $w, a<|w|<1$, and $a, \frac{1}{4}<a<1$, and $r=r(a)=$ $\frac{1}{5}(4 a-1)$

$$
T\left(r, f_{w}\right) \leq c \sup _{w \in D} \iint_{|z|<1} \frac{\left(1-|w|^{2}\right)\left(1-|z|^{2}\right)}{|1-\bar{w} z|^{2}}\left[f^{\#}(z)\right]^{2} d x d y \leq c c_{1}<+\infty
$$

where

$$
c_{1}=\sup _{w \in D} \iint_{|z|<1} \frac{\left(1-|w|^{2}\right)\left(1-|z|^{2}\right)}{|1-\bar{w} z|^{2}}\left[f^{\#}(z)\right]^{2} d x d y
$$

and $c_{1}<+\infty$ since $\mu_{f}$ is the Carleson measure (see [1], Lemma 3.3, p. 239).

For every $w \in D$ and any $r_{1}, 0<r_{1}<1$, the estimate

$$
\left(1-|w|^{2}\right) f^{\#}(w) \leq \frac{1}{r_{1}^{2}}\left(\exp 2 T\left(r_{1}, f_{w}\right)-1\right)^{1 / 2}
$$

is proved by S. Yamashita ([8], p. 193, the inequality (3.5)).

Combining (7) and (8), we obtain the inequality

$$
\left(1-|w|^{2}\right) f^{\#}(w) \leq \frac{1}{r^{2}}\left(\exp 2 c c_{1}-1\right)^{1 / 2}=M<+\infty
$$

valid for any $w, a<|w|<1$, and $r=r(a)$.

Since $f^{\#}(z)$ is a positive continuous function in $D$, we have for any $a_{1}$, $a<a_{1}<1$, and any $w,|w| \leq a_{1}$,

$$
\left(1-|w|^{2}\right) f^{\#}(w) \leq f^{\#}(w) \leq m<+\infty,
$$

where $m=\max _{|w| \leq a_{1}} f^{\#}(w)$.

Combining (9) and (10) we get

$$
\sup _{w \in D}\left(1-|w|^{2}\right) f^{\#}(w) \leq \max (M, m)<+\infty,
$$

that is, $f(z) \in N$. The Lemma is proved. 
Remark 1. Our Lemma essentially improves a result in [6], p. 42.

Remark 2. Let $f(z)$ be a holomorphic function in the disk $D$ and let $d \lambda_{f}(z)$ be the differential form $d \lambda_{f}(z)=\left(1-|z|^{2}\right)\left|f^{\prime}(z)\right|^{2} d x d y$. If, in the proof of the Lemma, we put $\left|f^{\prime}(z)\right|$ instead of $f^{\#}(z)$, we obtain a new proof of the following well-known result: If the measure $\lambda_{f}(z)$ for a holomorphic function $f(z)$ in $D$ is the Carleson measure, then $\lim \sup _{|z| \rightarrow 1}(1-|z|)\left|f^{\prime}(z)\right|<+\infty$; i.e., it is a Bloch function (cf. for instance [7], p. 481).

5. Theorem 3. A meromorphic function $f(z)$ belongs to the class $\cup \mathrm{BC}$ if and only if $\mu_{f}$ is a Carleson measure.

This theorem immediately follows from Theorem 2 and the Lemma. only if

Corollary. A meromorphic function $f(z)$ belongs to the class $\cup \mathrm{BC}$ if and

$$
\sup _{w \in D} \iint_{|z|<1} \frac{\left(1-|w|^{2}\right)\left(1-|z|^{2}\right)}{|1-\bar{w} z|^{2}}\left[f^{\#}(z)\right]^{2} d x d y \leq c c_{1}<+\infty .
$$

6. Theorem 4. A meromorphic function $f(z)$ belongs to the class $\cup \mathrm{BC}$ if and only if

$$
\sup _{w \in D} \iint_{|z|<1} T\left(1, f_{z}\right)\left|\varphi_{w}^{\prime}(z)\right|^{2} d x d y<+\infty .
$$

This theorem follows from Theorem 3 and a result in [3], Theorem 4 .

7. A well-known result states that a holomorphic function $f(z)$ in $D$ belongs to the class BMOA if and only if the measure $\lambda_{f}, d \lambda_{f}(z)=\left(1-|z|^{2}\right)\left|f^{\prime}(z)\right|^{2} d x d y$, is the Carleson measure (see for instance [7], p. 481). We note that the proof of Theorem 3 presents a new proof of this result if in the proof of Theorem 3 one uses $\left|f^{\prime}(z)\right|$ instead of $f^{\#}(z)$ and the inequality (3.5.3) in [8], p. 194 instead of (8).

I wish to express my gratitude to V.I. Gavrilov and S. Yamashita for their valuable advice. 


\section{References}

[1] Garnet, B.J.: Bounded analytic functions. - Academic Press, 1981.

[2] Lehto, O., and K.I. VirtanEN: Boundary behaviour and normal meromorphic functions. - Acta Math. 97, 1957, 47-65.

[3] PavićEvić, Ž.: The Carleson measure, Ahlfors-Shimizu characteristic and classes BMO and BMOA. - Dokl. Akad. Nauk SSSR (to appear).

[4] Yamashita, S.: Functions of uniformly bounded characteristic. - Ann. Acad. Sci. Fenn. Ser. A I Math. 7, 1982, 349-367.

[5] Yamashita, S.: Normality and the Shimizu-Ahlfors characteristic function. - Ann. Acad. Sci. Fenn. Ser. A I Math. 11, 1986, 207-213.

[6] Yamashita, S.: Image area and functions of uniformly bounded characteristic. - Comment. Math. Univ. St. Paul. 34, 1985, 37-44.

[7] Yamashita, S.: Some unsolved problems on meromorphic functions of uniformly bounded characteristic. - Internat. J. Math. Math. Sci. 8, 1985, 477-482.

[8] Yamashita, S.: Estimates of the derivatives by the Shimizu-Ahlfors characteristic functions. - Comment. Math. Univ. St. Paul. 35, 1986, 189-199.

University of Titograd

Faculty of Mathematical Sciences

Cetinjski put b.b.

P.O. Box 176

81000 Titograd

Yugoslavia

Received 3 September 1990 